\title{
Synthesis of Findings, Current Investigations, and Future Directions: Operation Brain Trauma Therapy
}

\author{
Patrick M. Kochanek, ${ }^{1}$ Helen M. Bramlett, ${ }^{2,3}$ Deborah A. Shear, C. Edward Dixon, ${ }^{4}$ Stefania Mondello, \\ W. Dalton Dietrich, Ronald L. Hayes, Kevin K.W. Wang, Samuel M. Poloyac, Philip E. Empey, \\ John T. Povlishock, ${ }^{10}$ Andrea Mountney, Megan Browning, ${ }^{1}$ Ying Deng-Bryant, ${ }^{4}$ Hong Q. Yan, \\ Travis C. Jackson, ${ }^{1}$ Michael Catania, ${ }^{11}$ Olena Glushakova, ${ }^{11}$ Steven P. Richieri, ${ }^{11}$ and Frank C. Tortella ${ }^{4}$
}

\begin{abstract}
Operation Brain Trauma Therapy (OBTT) is a fully operational, rigorous, and productive multicenter, pre-clinical drug and circulating biomarker screening consortium for the field of traumatic brain injury (TBI). In this article, we synthesize the findings from the first five therapies tested by OBTT and discuss both the current work that is ongoing and potential future directions. Based on the results generated from the first five therapies tested within the exacting approach used by OBTT, four (nicotinamide, erythropoietin, cyclosporine A, and simvastatin) performed below or well below what was expected based on the published literature. OBTT has identified, however, the early post-TBI administration of levetiracetam as a promising agent and has advanced it to a gyrencephalic large animal model-fluid percussion injury in micropigs. The sixth and seventh therapies have just completed testing (glibenclamide and Kollidon VA 64), and an eighth drug (AER 271) is in testing. Incorporation of circulating brain injury biomarker assessments into these pre-clinical studies suggests considerable potential for diagnostic and theranostic utility of glial fibrillary acidic protein in pre-clinical studies. Given the failures in clinical translation of therapies in TBI, rigorous multicenter, pre-clinical approaches to therapeutic screening such as OBTT may be important for the ultimate translation of therapies to the human condition.
\end{abstract}

Key words: biomarker; controlled cortical impact; drug; fluid percussion; micropig; penetrating ballistic-like brain injury; pre-clinical modeling; rat; reproducibility; therapy; traumatic brain injury

\section{Introduction}

N THIS SERIES OF ARTICLES, ${ }^{1-7}$ we have reported on the design, establishment, and implementation of the Operation Brain Trauma Therapy (OBTT) pre-clinical therapy and biomarker screening consortium. We have presented the findings of the first five therapies that were evaluated — namely, nicotinamide, erythropoietin (EPO), cyclosporine A (CsA), simvastatin, and levetiracetam ${ }^{2-6}$ and reported on the performance of two biomarkers of brain injury, Ubiquitin carboxyl-terminal hydrolase-L1 (UCH-L1) and glial fibrillary acidic protein (GFAP) across the three rodent traumatic brain injury (TBI) models used in therapeutic screening.

As described in the individual articles, the design of the OBTT screening consortium featured three different TBI rat models (parasagittal fluid percussion injury [FPI], controlled cortical impact $[\mathrm{CCI}]$, and penetrating ballistic-like brain injury [PBBI]), a battery of established and conventional functional and histological outcomes, a careful and comprehensive approach to therapy selection, a literature-based approach to treatment protocol development that was implemented in an identical fashion across sites,

\footnotetext{
${ }^{1}$ Department of Critical Care Medicine, Safar Center for Resuscitation Research, University of Pittsburgh School of Medicine, Pittsburgh, Pennsylvania.

${ }^{2}$ Department of Neurological Surgery, The Miami Project to Cure Paralysis, Miller School of Medicine, University of Miami, Miami, Florida.

${ }^{3}$ Bruce W. Carter Department of Veterans Affairs Medical Center, Miami, Florida.

${ }^{4}$ Brain Trauma Neuroprotection/Neurorestoration, Center for Military Psychiatry and Neuroscience, Walter Reed Army Institute of Research, Silver Spring, Maryland.

${ }^{5}$ Department of Neurological Surgery, Brain Trauma Research Center, University of Pittsburgh School of Medicine, Pittsburgh, Pennsylvania.

${ }^{6}$ Department of Neurosciences, University of Messina, Messina, Italy.

${ }^{7}$ Center for Innovative Research, Center for Neuroproteomics and Biomarkers Research, Banyan Biomarkers, Inc., Alachua, Florida.

${ }^{8}$ Center of Neuroproteomics and Biomarkers Research, Department of Psychiatry and Neuroscience, University of Florida. Gainesville, Florida.

${ }^{9}$ Center for Pharmaceutical Sciences, University of Pittsburgh School of Pharmacy, Pittsburgh, Pennsylvania.

${ }^{10}$ Department of Anatomy and Neurobiology, Virginia Commonwealth University, Richmond, Virginia.

${ }^{11}$ Banyan Biomarkers, Alachua, Florida.
} 
and a highly rigorous approach to therapy and biomarker assessments.

In this concluding article, we synthesize the key findings from this initial work by the consortium, provide insight into the ongoing investigations, and formulate potential avenues for future directions.

\section{Summary and Synthesis of Findings}

\section{Strategy}

Crafting and establishing a multicenter consortium approach to pre-clinical therapy development represented a novel initiative for the field of TBI; however, we were fortunate that there was some precedent on which to base our overall plan for therapy testingnamely, the Multicenter Animal Spinal Cord Injury Study (MASCIS) from the late 1990s. ${ }^{8,9}$ As discussed in the introductory article in this issue of the journal, ${ }^{1}$ in MASCIS, there was an attempt to standardize the approach to therapy testing by using a single model of spinal cord injury across all sites. Regrettably, that led to major challenges in reproducibility and differential complications across the sites, and ultimately a large number of therapies were not evaluated. The failure to reproduce the purported efficacy of the bellwether agent methylprednisolone by the MASCIS consortium also blunted momentum.

Building on that valuable knowledge, in OBTT we chose to use a range of models that were already established and being actively used for therapy testing at each site, and instead, rigorously standardized the approach to treatment across sites. We believe that that decision contributed critically to the success of rapidly launching OBTT and facilitated the prompt and ongoing screening of multiple therapies, as presented in this issue. Similarly, we chose to use outcomes that were already established at each site-rather than attempting to mandate use of identical outcomes across sitesoutcomes that might have needed to be established at a given site. Thus, as was evident from the reports on each therapy, the motor tasks used, for example, differed across sites.

This approach, however, allowed investigators to use established injury levels at each site and immediately begin therapy screening, rather than try to define an injury level that produced usable deficits on outcomes that may have neither been established nor routinely used in their model and/or center. We were fortunate that the Morris water maze (MWM) was already established and routinely used at each site, and thus a key cognitive outcome tool was able to be readily incorporated into the OBTT scoring matrix. We were also able to establish an approach to histopathological screening that was readily applicable with either no or only minimal modifications at each site. We believe that those initial decisions were essential to the ability of OBTT to launch and produce meaningful data in a prompt and successful manner.

We also chose to weigh cognitive outcome as having the greatest impact on perceived therapy success in our scoring matrix. That decision was in some ways arbitrary-although the importance of cognitive outcome as a therapeutic target is certainly not questioned in the setting of severe TBI. ${ }^{10}$

Although we believe that the scoring matrix that was developed is reasonable, we recognize, however, that the exact weighting of the various outcomes across the sites was defined simply by the consensus of our collective investigative team and might not be optimal in some applications. For example, a therapy targeting brain edema might be important to preventing herniation and/or the need for decompressive craniectomy in humans with severe TBI, but that might not be readily reflected in improved cognitive outcome in our models - each of which includes a craniotomy as part of its design. As with any pre-clinical data, these and other related factors should be carefully vetted when considering any therapy for clinical translation.

\section{Models}

Another goal of OBTT was to use a broad menu of TBI models in rats in primary screening in an attempt to specifically address the well-recognized issue of heterogeneity of TBI as a roadblock to successful therapy development. ${ }^{11,12}$ It became clear rapidly that the three models that we selected achieved that goal and provided both a wide range of injury severity, behavioral deficits, neuropathological alterations, biomarker levels, and responses to therapy.

We also believe that OBTT provided a heretofore unexplored direct comparison between three rodent models commonly used in the field of pre-clinical TBI research-providing unique insight for the field. For example, visual comparison of the TBI vehicle groups in the pooled outcomes figures in any of the individual treatment articles in this special issue ${ }^{2-6}$ immediately reveals the marked differences in outcomes between models. Specifically, examination of the pooled analysis graphs in each of the aforementioned manuscripts comparing lesion volume at 21 days after injury across models demonstrates the relatively small focal lesion in the parasagittal FPI model in rats compared with either CCI or PBBI, and the similarities between CCI and PBBI for this outcome with, in general, slightly greater lesion volume in PBBI versus CCI.

Careful consideration of the methods also indicates that the difference in the size of the focal lesion in FPI versus either CCI or PBBI is even greater than suggested by those figures, given the fact that lesion volume is normalized to cortical volume in FPI, while it is normalized to hemispheric volume (a much larger denominator) in both CCI and PBBI. These types of comparisons are, we believe, unique in our field and also help in explaining the differences between models in the observed behavioral outcomes such as average latency to find the hidden platform in the MWM paradigm-which in general were much shorter in the FPI model versus either CCI or PBBI. Surprisingly, despite what would amount to apparently much smaller therapeutic targets in FPI, in general, the greatest number of therapeutic benefits were shown in FPI and CCI.

Issues such as severity or manipulability of the insult, other facets of the type of injury produced, and the ability of a chosen therapy to target a given injury substrate may be paramount to being able to demonstrate therapeutic efficacy. This issue is discussed in greater detail later in this article. In any case, given that the same rigor was applied across sites, we believe that OBTT provides special insight for model comparisons in this regard. Given the primary goal of OBTT to identify the most promising therapies for clinical evaluation in severe TBI, we have only scratched the surface related to data analysis on cross model comparisons from the myriad results of OBTT in this regard.

\section{Biomarkers}

We would be remiss to not mention the fact that in its original form, OBTT was proposed purely as a drug screening consortium. The concept of incorporating circulating biomarker assessments into the program came at the request of the reviewers of our original OBTT grant submission to the U.S. Department of Defense. We are grateful to those reviewers for that suggestion and believe that the incorporation of circulating biomarkers of brain injury into the work of our consortium has generated some remarkable results and has added considerable richness to our findings. In addition, by using biomarkers that are currently in clinical trials, we believe that the 
results generated thus far by OBTT could provide insight on biomarker use and interpretation germane to clinical investigationswhere the exact nature of the injury is often unclear or complex.

A number of very interesting findings were generated by OBTT based on our biomarker results-we will highlight three of them in this summary article. First, our data strongly suggest circulating levels of GFAP represent an excellent biomarker of brain injury for pre-clinical investigations, and that this is likely to be the case in the clinical arena. As clearly demonstrated in the article in this issue by Mondello and associates, ${ }^{7}$ GFAP levels were not only reproducibly increased at 4 and $24 \mathrm{~h}$ after injury across models comparing the TBI vehicle and sham groups, from study to study, the $24 \mathrm{~h}$ levels were correlated strongly with histological outcomes and in some cases, with behavioral outcomes.

These correlations were seen across models, and were truly exceptional in the CCI model for the relationships between $24 \mathrm{~h}$ GFAP levels and $21 \mathrm{~d}$ lesion volume and hemispheric tissue loss. UCH-L1 did not perform as well, although it might merit evaluation at earlier time points after injury, given its short half-life and rapid appearance in serum after TBI in humans. ${ }^{13}$ The ability to use time points, however, with broad clinical relevance such as $4 \mathrm{~h}$ and $24 \mathrm{~h}$, as shown with GFAP, is attractive for a biomarker.

Second, the biomarker data revealed some very surprising crossmodel findings that may provide special insight into pre-clinical and clinical data interpretation. One of the most interesting in this regard was the fact that despite the modest lesion size in FPI, serum GFAP levels were higher at $4 \mathrm{~h}$ after injury than in the PBBI model, as assessed when comparing TBI vehicle groups. By $24 \mathrm{~h}$, this finding had reversed-although serum GFAP levels were still only modestly greater in PBBI than in FPI despite hugely different amounts of tissue loss.

A number of factors could be involved. For example, cerebral blood flow is likely much more well preserved in the FPI model and thus a larger volume of injured tissue may still be well perfused in FPI compared with PBBI, where a large area of brain is rapidly and severely damaged in the experimental ballistic tract by the PBBI mechanism that mimics what is seen in the clinical setting of a ballistic tract after a penetrating brain injury. Other factors could be involved such as lesion location and differences in blood-brain barrier permeability (in regions that remain perfused).

One other emerging area of biomarker research relates to the role of the glymphatic system on movement of parenchymal biomarkers to the circulation, ${ }^{14}$ and differences between models on the impact of injury on that pathway could also be involved. In those studies, the impact of disruption of the glymphatic system was shown at $18 \mathrm{~h}$ after TBI. Differences between models that we observed as rapid as $4 \mathrm{~h}$ after injury, however, may suggest more direct transfer of biomarkers into the circulation. Further study is needed in this regard.

Third, we were very pleased that circulating GFAP levels at $24 \mathrm{~h}$ after injury in the CCI model predicted a benefit of most efficacious therapy tested to date in OBTT (levetiracetam) on ultimate hemispheric tissue loss at 21 days after injury. The benefit of levetiracetam in pre-clinical TBI was suggested in the initial work of Wang and colleagues, ${ }^{15}$ and thus OBTT was able to replicate, in many ways, that positive effect.

It was also interesting to see that the increase in lesion volume seen with simvastatin treatment in the FPI model was also reflected in an increase in serum GFAP levels at $24 \mathrm{~h}$-although this was not as clearly delineated as in the case with levetiracetam, because both the low and high dose treatments with simvastatin increased serum GFAP, while only the high dose exacerbated tissue loss. In any case, the potential theranostic utility of GFAP is exciting, particularly given that $24 \mathrm{~h}$ circulating levels are predicting long-term histology at $21 \mathrm{~d}$.

One could also argue that although not statistically significant, a similar trend of reduced GFAP levels at $24 \mathrm{~h}$ after injury predicting a reduction in hemispheric tissue loss was seen in the CCI model with the only other drug that significantly affected this histological outcome parameter in OBTT - namely high dose nicotinamide. ${ }^{7}$ Histological benefit was suggested in the CCI model by the work of Hoane and coworkers, ${ }^{16}$ which served as the basis of the treatment protocol used for nicotinamide by OBTT. A larger sample size would be needed to appropriately test the utility of GFAP to predict tissue loss in the CCI model with nicotinamide-but it is clear that this is worthy of additional exploration.

Although both levetiracetam and nicotinamide reduced hemispheric tissue loss in CCI, neither drug produced a statistically significant reduction in contusion volume in the CCI model. Hemispheric tissue loss in CCI comprises both the contusional volume loss and an additional volume of tissue lost outside of the contusion in the impacted hemisphere-an amount that often is similar in magnitude to the tissue volume in the contusion proper. It might be that this "occult" or "silent" volume loss is more therapeutically manipulable than the parenchyma directly impacted by primary injury located in the contusion proper. It will be interesting to follow this parameter in OBTT to determine whether other drugs can successfully reduce contusion volume versus hemispheric tissue loss in CCI and the other models being used.

Finally, as previously discussed, ${ }^{17}$ hemispheric tissue loss often better correlates with MWM latency than lesion volume in CCI.

\section{Therapies}

In general, within the exacting approach used by OBTT, most of the therapies performed below or well below what was expected based on the published literature. One of the major goals of OBTT is to define a therapy that is highly effective across all three models, in an attempt to address the heterogeneity of TBI that has been suggested to be vital to successful pre-clinical drug development to mimic the clinical condition. ${ }^{11,18}$

None of the first five therapies proved beneficial across all three screening models, although levetiracetam showed beneficial effects on multiple outcomes in both the parasagittal FPI model and CCI (Fig. 1). In addition, surprisingly, it was the only therapy to show beneficial effects on cognitive outcome in any of the models. Remarkably, its tissue sparing effect in CCI seen at 21 days after injury was predicted theranostically by $24 \mathrm{~h}$ blood GFAP levelsan exciting finding in the field of TBI biomarker research.

Modest and relatively sporadic beneficial effects were seen for nicotinamide (at the highest dose) and simvastatin (on motor function). A complete lack of benefit in any model was also quite surprising to see with EPO_which had $>20$ articles supporting its use specifically in pre-clinical TBI and many other supportive studies across other brain injury models. ${ }^{3}$ Supporting our findings, however, EPO failed to demonstrate benefit in a recent high-quality single center trial in $\mathrm{TBI}^{19}$ and similarly demonstrated a suggestion toward a detrimental effect in clinical stroke. ${ }^{20}$ Effects of CsA in OBTT were complex and highly model dependent-with some modest benefits in the FPI model-the mildest injury used for therapy screening in OBTT, but lack of benefit in CCI (and some toxicity) and deleterious effects in PBBI, the most severe injury model.

Some thoughts on why only levetiracetam met the performance standard suggested in the literature whereas these other seemingly promising therapies failed to show benefit in OBTT are provided in 


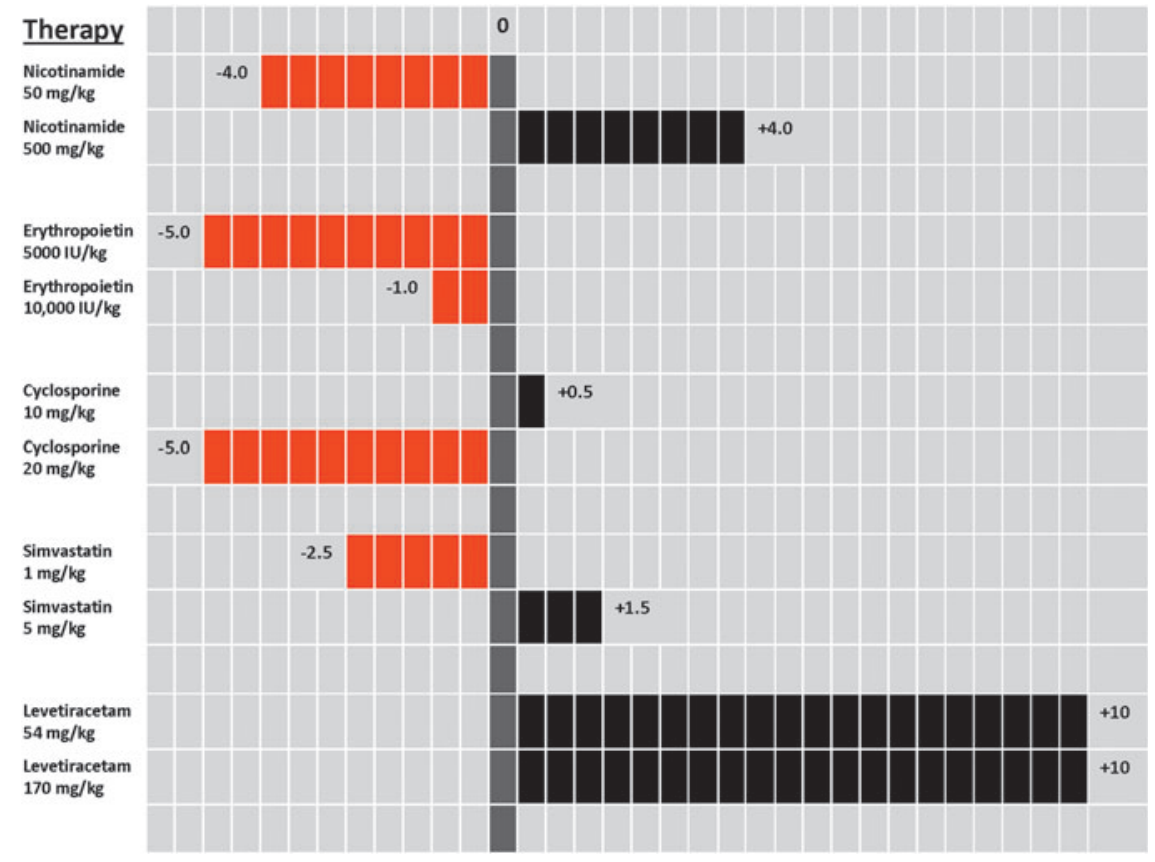

FIG. 1. Graphic representation of the overall scores from the Operation Brain Trauma Therapy (OBTT) scoring matrices generated from testing of each of the first five therapies evaluated across three rat models (parasagittal fluid percussion injury, controlled cortical impact, and penetrating ballistic-like brain injury). Note that for each drug, two doses were tested. Specifics of the dosing are provided in each of the treatment articles in this issue of the journal. ${ }^{2-6}$ Scores depicted in red indicate negative effects, while those in black indicate an overall positive effect. In general, most of the therapies underperformed relative to the published literature. Levetiracetam, however, was the most promising drug tested, was the only drug that showed strong effects on cognitive outcome in any model, and had no deleterious effects that generated negative points in any of the models. Levetiracetam is currently being evaluated in a micropig model within OBTT.

the sections below addressing (1) limitations of OBTT versus failure of reproducibility, (2) seeking a therapy that crosses models versus model specific therapy, and (3) translation of brain specific versus broader mechanism-targeting therapies.

Limitations of OBTT versus failure of reproducibility. One possible reason for the limited therapeutic success in OBTT is that there are many limitations to the approaches taken by OBTT. It is, thus, not OBTT's position to serve as a bully pulpit with regard to formulating impressions on the rigor of previous pre-clinical research or to forcefully shape the translational potential of the preclinical TBI arena and/or dismiss other results.

Some of the limitations of OBTT are obvious. OBTT does not study mechanism and thus did not demonstrate that the drugs and/or doses used affected the desired mechanistic target or targets. OBTT also screened only two doses of each therapy and performed studies in only a single injury level in each model. Although OBTT based its treatment protocols on the published literature as much as possible, given our goal of having a protocol that could have relevance to combat casualty care and be readily translated to a clinical trial, we often chose the intravenous rather than intraperitoneal route for drug administration. That likely changed drug kinetics in several cases and may have impacted efficacy and/or toxicity, as discussed later.

Dosing regimens different from those used thus might produce different results. This could be particularly true in OBTT given the fact that, depending on the drug, the optimal dose could vary across models. For example, this might in part be reflected in the highly variable effects of CsA in OBTT. CsA is a drug with a narrow therapeutic index that has complicated distribution and elimination kinetics that are altered after brain injury. It also has limited bloodbrain barrier permeability, and brain levels that are achieved likely vary greatly with model severity - and could produce a spectrum of benefit versus harm. ${ }^{4}$ Supporting this concept, levetiracetam-a drug with simple pharmacokinetics, excellent blood-brain barrier penetration, and a very large margin of safety-was the most successful.

Within OBTT for CsA, we observed some modest benefit in the mildest model (FPI) but substantial toxicity in the most severe insult (PBBI). To select the best possible doses, we have sought in many cases the specific input from our team members in the University of Pittsburgh School of Pharmacy (SMP and PEE), and in some cases have measured serum levels when there is limited and/ or equivocal literature support. To optimize clinical translation of the effective therapies, future studies should aim at not only evaluating the dose mediating improved outcomes but also assess the concentration required in the brain to mediate that observed effect.

As mentioned previously, there is also another possible issue related to drug administration and dosing that could impact reproducibility of treatment effects when compared with the existing pre-clinical literature by OBTT. In general, OBTT sought to use the intravenous route for drug administration, given the likelihood of the need for that route in the setting of combat casualty care and/or severe TBI in the civilian setting. Often the majority of studies in the published literature in rodent models, however, involved intraperitoneal drug administration where systemic drug bioavailability may be variable or reduced. Injury can also influence drug metabolism, further complicating dosing.

Finally, as discussed previously, the injury levels in each of the models in OBTT did not always produce an optimal therapeutic 
target for each therapy and a certain amount of "wobble" in the models was observed given the desire to produce behavioral deficits that were potentially manipulable by the treatments that were being screened. This may have underpowered some studies, particularly in FPI. Consistent with this hypothesis, the only model that produced a robust MWM latency target in every study was PBBI, yet that was the model that demonstrated the fewest therapeutic successes. This fact epitomizes the challenges faced when screening drugs across multiple models.

Thus, we appreciate the fact that despite the strong and carefully constructed methodologic rigor of OBTT on many fronts, pharmacologic rigor may have fallen short, particularly with respect to replicating specific previous literature reports. Other limitations inherent to the approach taken by OBTT are possible and some were discussed in the other articles in this special issue of the journal.

(1). Rigor and reproducibility. One alternative possible explanation for the performance of therapies in OBTT at a level below or well below what was expected based on the published literature is that the literature is inflated. Recently, inadequate rigor in pre-clinical research has been identified as an important potential contributor to the failure of clinical trials. This was brought to light in a landmark article by Begley and Ellis ${ }^{21}$ and Begley and Ioannidis ${ }^{22}$ that called into question pre-clinical research in the field of cancer therapy development when the Hematology and Oncology Department at the biotechnology firm Amgen tried to confirm published findings from 53 articles that were published in prestigious high impact basic science journals.

By the investigator's criteria, only $11 \%$ of the studies were able to be reproduced. This reproducibility assessment was performed because, mirroring TBI, randomized controlled clinical trials in the field of oncology were stated to have extremely high failure rates.

It is interesting that in several cases in OBTT, we based our selection on as many as 10-20 supportive published articles in preclinical TBI models, and in some cases we used the same or a similar dosing regimen suggested in some of the reports within a given body of work. This did not even take into account reports in other pre-clinical models such as stroke. Ironically, the most successful therapy in OBTT (levetiracetam) had only a single supportive paper in pre-clinical TBI at the time it was selected for screening, but it had other favorable characteristics including a purported brain-specific mechanism of action, a chemical structure with proven blood-brain barrier permeability with an ability to achieve therapeutically relevant concentrations in the brain, sporadic clinical use in TBI that was already ongoing, and a robust safety record supporting its selection. The rationale behind the selection of levetiracetam versus other potential therapies is discussed in greater detail later.

The role of the aforementioned failure of reproducibility on the findings of OBTT remains unclear, but reproducibility of published pre-clinical studies was not the stated goal of OBTT. Rather, we sought to use the published pre-clinical literature to aid in (1) selecting potential therapies, (2) formulating a pharmacological approach that was as clinically relevant as possible given the available literature, (3) screening those therapies at two doses across three very different rat models of TBI, and (4) seeking breakthrough effects in either one or multiple models across the consortium. This approach involved difficult compromises often related to issues relevant to pharmacology.

Another factor to consider is that unlike in vitro basic research, where it might be relatively straightforward to reproduce findings, pre-clinical TBI has always placed high value on behavioral outcomes and neuropathology at relatively long-term outcome time points. Clearly, reproducing those types of studies is demanding and expensive, and quite a high bar. This suggests that approaches such as OBTT (or others with a multicenter strategy with a high level of rigor) could be quite valuable to help direct the field toward the strongest possible candidates - using the established literature more as a repository of "clues" rather than as a stronger verdict on a given therapy.

Ramping up the rigor in all of our individual laboratories is also logical as suggested in recent publications in the fields of stroke, spinal cord injury, and central nervous system (CNS) injury ${ }^{23-25}$ and in the recent article on common data elements in pre-clinical $\mathrm{TBI}^{26}{ }^{26}$ Specific recommendations include carefully addressing issues such as group randomization, study blinding, sample size analysis, appropriate statistical approach, and transparency with regard to conflict of interest, among other issues, along with the important recommendation to reproduce the work before publication. With regard to increasing pharmacologic rigor, directly measuring dose-concentration relationships on mechanism and outcomes could be helpful.

Finally, in our current state of knowledge, it is very difficult if not impossible to define a treatment effect on either MWM or histology in a pre-clinical study that is linked to a known clinical outcome success.

(2) A therapy that crosses models versus model specific therapy. As discussed previously, one factor believed to be important to the failed translation of therapies from the pre-clinical to the clinical arena is the fact that clinical TBI is extremely heterogeneous. Thus, it is believed that the chances for successful translation might be increased if a drug was shown to have benefits when tested across multiple models of TBI. That possibility was one of the premises on which the design of OBTT was based.

To date, no therapy tested by OBTT has shown robust benefit across all three primary screening models-particularly on longterm cognitive outcome. To date, only levetiracetam has shown fairly robust benefit, but that is limited to two of the three models and does not include robust benefit on cognitive outcome in both models. An alternative conclusion of the findings of OBTT to date may thus be that a personalized or precision medicine (model specific) approach to the treatment of TBI is needed.

This is certainly a real possibility and might be even more likely than suspected from the data provided thus far in this issue given the fact that in OBTT, we limited our modeling to clinical analogs of severe TBI or possibly (given the injury level seen in parasagittal FPI) moderate or moderate to severe TBI. Broadening the clinical target to include the full spectrum of injury severity suggests that the need for a personalized medicine approach for clinical translation is even more likely. This would suggest that clinical investigators should consider testing therapies in patients that mimic the models that demonstrate the greatest beneficial effects of a given therapy. Those studies should also measure drug exposure and theranostic markers if available. OBTT can thus contribute special insight into such an approach given that the three models that are being used are quite different.

We did not, however, include a diffuse closed head injury model, a blast TBI model, or a TBI plus polytrauma model (among others) in our OBTT screening approach, and there is substantial evidence that various aspects of the secondary injury mechanisms involved in the exacerbation of damage are unique. ${ }^{27-33}$ An even broader model representation for OBTT might thus be desirable for future 
investigations. Given that screening of only five drugs is reported, however, and that only two additional drugs have completed studies (along with an eighth drug currently in testing), it would be, in our opinion, incorrect to come to premature closure on the ability to identify a highly robust therapy that crosses all three models.

It is also possible that for any of the drugs tested, the optimal dose to show benefit would differ between models tested in OBTT. For therapies targeting acute neuroprotection, however, clinical dosing has, to our knowledge, not been titrated to severity of injury in randomized controlled trials (RCTs) - so from a clinical translation standpoint, we believe that the goal of OBTT to demonstrate efficacy of a therapy across the three models (despite their great differences in severity) at a given dose is justified. Even in the meticulously executed recent trial of progesterone by Wright and colleagues $^{34}$ where patients with both moderate and severe TBI were randomized, for example, all patients received the same dose.

Clinical trials invariably test the therapy in an RCT at one or possibly two doses as used in our OBTT study design. Thus, in an attempt to maximize clinical translation, we may in fact have underestimated the potential efficacy of individual drugs in individual models using the screening strategy taken by our consortium. Nevertheless, we believe that this represents a strength rather than a liability for OBTT. If a therapy indeed is robust across all models at the same dose, it would greatly strengthen the chances of successful clinical translation.

Thus, there are both strengths and weaknesses to the approach used by OBTT. We remain optimistic that a more potent and robust therapy that crosses models will be identified by our approach.

(3) Brain specific versus broader mechanism-targeting therapies. Another intriguing finding based on the results of the first five therapies screened by OBTT is the fact that the drug that has demonstrated the most benefit (levetiracetam) is the only one that was drug specifically designed/developed to treat a pathophysiological process in the brain-namely, seizures. Given the fact that there is an empiric use of a number of therapies in neurocritical care in the treatment of patients with severe TBI-such as anticonvulsants, analgesics, and sedatives, and hyperosmolar agents, among others-there has, in general, been a focus in the pre-clinical literature on unique therapies that target broader secondary injury mechanisms (that operate both within and outside of the CNS) such as apoptosis, mitochondrial failure, oxidative stress, proteolysis, autophagy, and/or other pathways.

The findings of OBTT presented, however, suggest that such an approach, although tantalizing for identifying a unique breakthrough therapy, may actually have a lower chance of success than exploring more highly brain specific mechanisms. Bullock and associates $^{35}$ and Tolias and Bullock ${ }^{36}$ have long suggested that a major limitation of TBI research in clinical translation has been in the area of the clinical assessment of brain pharmacokinetics and pharmacodynamics of therapies. Issues such as robust blood-brain barrier permeability and lack of neurotoxicity, for example, may be paramount to success and dwarf other mechanistic factors-which are often highlighted in pre-clinical reports.

There are many brain specific targets in the secondary injury cascade such as excitotoxicity, spreading depression, axonal injury, glial alterations, and loss of trophic support, and these may represent important targets using drugs specifically designed as CNS targeting therapies. One could argue, alternatively, that demonstrating benefit from a drug such as levetiracetam by OBTT has limited value. Rats, unlike patients, do not routinely receive anticonvulsants in the acute phase after severe TBI, and thus it might remain difficult to demonstrate a clinical benefit of levetiracetam in an RCT. Studies by Darrah and coworkers ${ }^{37}$ suggest, however, that unlike levetiracetam, phenytoin demonstrates deleterious effects in the CCI model in rats, and given the fact that both phenytoin and levetiracetam are used clinically, an advantage might be able to be shown in a clinical RCT.

In addition, one of the most interesting findings in our studies with levetiracetam is that it improved multiple long-term outcomes despite the fact that it was administered as a single bolus at $15 \mathrm{~min}$ after injury. As discussed earlier in this special issue, ${ }^{6}$ that may suggest beneficial effects on mechanisms other than seizures. In any case, we believe that the findings to date in OBTT suggest that additional drugs that were specifically designed for use in the CNS should be explored.

\section{Current Investigations}

Investigations are ongoing in OBTT. Given the success of levetiracetam, it has been advanced to testing in a gyrencephalic animal model-namely, FPI in micropigs. It is noteworthy that the outcomes in that model include assessments of axonal injury and cerebrovascular responsivity, along with serum and tissue biomarkers (GFAP, UCH-L1, and ionized calcium-binding adapter molecule 1 [IBA-1]). This will allow both a direct comparison of rodent and large animal response to a promising therapy, but it will also provide some unique therapeutic targets that are not part of therapeutic screening in the rodent models in OBTT. This crossspecies investigation within OBTT is exciting.

With regard to therapeutic screening in the rat model, currently studies have been completed and data analysis ongoing on two additional drugs — namely, glyburide and Kollidon VA64. In addition, with research support provided by the U.S. Department of Defense based on performance of the OBTT consortium and on the desire to test additional therapies that may be somewhat earlier in development and/or proprietary, a grant titled OBTT-Extended Studies (OBTT-ES) is currently supporting assessment by our consortium of the aquaporin 4 antagonist (AER-271); the OBTT-ES program just launched.

In addition, exploratory dosing studies and protocol planning are under way for minocycline and amantadine, which will likely be tested by this year by OBTT, along with other agents. These agents are specifically targeting mechanisms such as cerebral edema, ${ }^{38}$ neuroinflammation, ${ }^{39,40}$ and cognitive enhancement, ${ }^{41,42}$ which have not been explored to a significant extent thus far by our consortium and are logical candidate mechanisms. We are particularly interested in testing amantadine given the fact that it has shown success in a RCT in the setting of severe TBI in humans ${ }^{43}$ and that work was based on the seminal study by Dixon and colleagues $^{41}$ in the CCI model using that therapy.

\section{Future Directions}

\section{Therapy}

One of the key questions in the search for new therapies for TBI that future studies by OBTT and/or other similar initiatives should address is whether the most fruitful path lies in using therapies to prevent the evolution of secondary damage or manipulate the remaining circuitry. ${ }^{44}$ It is certainly logical to pursue both strategies, with the ultimate goal of new therapy development on both fronts. Nevertheless, it is not clear which approach is most likely to lead to major improvements in outcome.

Another question that is often raised at presentations of the work of OBTT is whether or not the consortium is considering 
combination therapy. It is likely that combination therapy will ultimately be needed to maximize outcomes in pre-clinical models and patients with severe TBI; however, we believe that we are at an early point in the evolution of the consortium approach to preclinical therapy development and have sought to first carefully evaluate individual therapies, generating a body of individual comparisons of therapies that can-at the least—serve as a future road map. Indeed, key basic elements such as drug levels and dose response deserve to be more carefully and thoroughly evaluated, even in studies of individual therapies.

Thus far in OBTT, we have focused on assessment of drugs, but we recognize that approaches such as cellular therapies ${ }^{45,46}$ or other nonpharmacological therapies ${ }^{47}$ should be considered. Issues such as prevention or resilience are also of potential importance particularly when considering military relevance, and thus a pre-treatment approach might also be worthy of consideration for certain therapies. Given the goal of identifying a robust therapy for a RCT in the setting of severe TBI in civilians (which would be necessary for ultimate translation), however, we have logically focused on post-TBI drug administration.

Finally, we have focused most of our efforts on acute administration of therapies, given the premise that delay in the onset of treatment for most mechanisms reduces therapeutic efficacy. Given the role of mechanisms such as subacute neuroinflammatory cascades ${ }^{48}$ however, additional consideration might be given to the prolonged administration of a given therapy. Thus far, prolonged therapy was tested for only one agent by OBTT-namely, simvastatin. Unfortunately, we did not see robust benefit in any of the models using simvastatin despite prolonged treatment. Other agents potentially targeting neuroinflammation and/or neurodegeneration related pathways might be more successful and deserve exploration.

\section{Biomarkers}

(1) Use and optimization of current biomarkers. As discussed previously, using a protocol that included blood sampling at $4 \mathrm{~h}, 24 \mathrm{~h}$, and 21 days after TBI, GFAP outperformed UCH-L1 both as a diagnostic and theranostic in the initial five therapeutic screening studies in OBTT. Given its short half-life, ${ }^{13}$ it is possible that the performance of UCH-L1 could be improved with sampling earlier after TBI. We have recently added a $1 \mathrm{~h}$ sampling point to the protocol and are currently reexamining how UCH-L1 performs across our models. In addition, assays for GFAP and UCH-L1 are currently in development, and testing for work in the micropig model and serial blood sampling is being performed in that model along with an assessment of correlation with brain tissue levels of both markers as assessed by immunohistochemistry.

(2) Additional biomarkers in development. Using an assay developed at the University of Florida, we are beginning to explore the potential utility of serum levels of IBA-1 as a TBI biomarker. These studies have been initiated in the micropig model, and if successful, there is a plan to add this biomarker to the rat panel. This is a very logical biomarker to pursue given the robust and sustained microglial response seen across our models after TBI including both rat and micropig. Several other circulating biomarkers are being considered for assessment.

\section{Modeling}

Given the fact that we have shown feasibility of the OBTT consortium concept, a number of potential avenues for expansion and/or modification can be raised. With the emergence of the importance of mild TBI and mild repetitive TBI, inclusion of a representative model of these insults would seem to be an additional and valuable opportunity. This was not considered to be feasible when OBTT was planned and launched, because at that time, there were few established pre-clinical models of mild TBI. One concern with regard to mild TBI and the OBTT concept is the fact that although new models are emerging, only a limited number of therapies have been tested in pre-clinical models of mild TBI; thus the basis for therapeutic testing would likely still rest on experience in pre-clinical models of severe TBI.

OBTT includes an important model of TBI that is highly relevant to combat casualty care-namely, PBBI. In future work, however, some consideration might be given to inclusion of a blast TBI model, where some therapy screening has been performed. ${ }^{49}$ At the least, the most promising agents identified by our screening approach taken in OBTT-in our opinion — should be tested in blast TBI models using the treatment protocols identified as successful by our consortium.

A progressive encephalopathic process (characterized by progressive tissue loss and prolonged behavioral deficits) over as long as 1 year has been demonstrated in pre-clinical models of TBI, ${ }^{50-52}$ including studies in some of the specific models in use in OBTT. Consideration thus might be warranted for the use of an OBTT-like approach to the assessment of the impact of acute and/or chronic treatment on longer-term outcomes. The emerging importance of the link between TBI and various neurodegenerative diseases suggests that such an approach could be quite important. Given the limited experience with this approach even within individual laboratories, the labor intensive nature of these studies, and their high cost, however, careful planning would be essential. In that regard, the lessons learned from the past and ongoing OBTT studies by OBTT would be important to guiding that work.

\section{Conclusions}

OBTT is an established, fully operational, rigorous, and highly productive multicenter, pre-clinical drug and circulating biomarker screening consortium. Based on the results generated from the first five therapies evaluated, within the exacting approach used by OBTT, four (nicotinamide, erythropoietin, cyclosporine A, and simvastatin) of the five therapies performed below or well below what was expected based on the published literature. OBTT, however, has identified the early post-TBI administration of levetiracetam as a promising agent and has advanced it to a FPI model in micropigs. Two additional therapies (the sixth and seventh) have just completed testing (glibenclamide and Kollidon VA 64) with results on those agents beginning to emerge, and an eighth drug (AER 271) is currently in testing.

Incorporation of circulating biomarker assessments into these pre-clinical studies has suggested potential for diagnostic and theranostic utility of GFAP - which could potentially simplify and/ or aid in initial screening of TBI therapies in pre-clinical models. Additional validation of the use of GFAP as a theranostic tool in pre-clinical work is needed, however, both in future studies in OBTT and outside of the OBTT consortium. Given the concerns related to what has been described as a reproducibility crisis in basic and pre-clinical science across disciplines, and the many failures in clinical translation of therapies specifically in TBI, rigorous multicenter pre-clinical approaches to therapeutic screening as carried out by OBTT may be important for the ultimate translation of therapies to the human condition. 


\section{Acknowledgment}

We are grateful to the U.S. Department of Defense grants WH81XWH-10-1-0623 and WH81XWH-14-2-0018 for generous support. We would like to thank Col. Dallas Hack for his strong support of our program, his vision for TBI research, and his scientific input. We also thank Dr. Kenneth Curley for his administrative support and his many contributions to identification of emerging therapies. We thank Dr. Brenda Bart-Knauer for her support of our program and her administrative assistance. We thank Linda Ryan for administrative support with budgetary issues across the consortium, Fran Mistrick for other administrative and coordinating support, and Marci Provins and Natalie Nieman for assistance with manuscript preparation, and Vincent Vagni for assistance with Figure preparation. We thank Rebecca Pedersen, Justin Sun, Ofelia Furones-Alonso, Milton Martinez, Juliana Sanchez-Molano, William Moreno, Ryan Treu, Jessie Truettner, Michelle Ma, Jeremy Henchir, and Keri Feldman for outstanding technical support in the individual TBI models across the consortium.

This material has been reviewed by the Walter Reed Army Institute of Research. There is no objection to its presentation and/or publication. The opinions or assertions contained herein are the private views of the authors, and are not to be construed as official, or as reflecting true views of Department of the Army or Department of Defense.

\section{Author Disclosure Statement}

Drs. Hayes and Mr. Richieri own stock and are both officers of Banyan Biomarkers Inc. Drs. Hayes and Catania, Mr. Richieri, and Ms. Glushakova are employees and receive salaries and stock options from Banyan Biomarkers Inc. Dr. Wang is a former employee of Banyan Biomarkers Inc. and owns stock. Drs. Hayes and Wang also receive royalties from licensing fees and, as such, all of these individuals may benefit financially as a result of the outcomes of this research or work reported in this publication. For the remaining authors, No competing financial interests exist

\section{References}

1. Kochanek, P.M., Bramlett, H.M., Dixon, C.E., Shear, D.A., Dietrich, W.D., Schmid, K.E., Mondello, S., Wang, K.K., Hayes, R.L., Povlishock, J.T., and Tortella, F.C. (2016). Approach to modeling, therapy evaluation, drug selection, and biomarker assessments, for a multicenter pre-clinical drug screening consortium for acute therapies in severe traumatic brain injury: Operation brain trauma therapy. J. Neurotrauma 33, 513-522.

2. Shear, D.A., Dixon, C.E., Bramlett, H.M., Mondello, S., Dietrich, W.D., Deng-Bryant, Y., Schmid, K.E., Wang, K.K., Hayes, R.L., Povlishock, J.T., Kochanek, P.M., and Tortella, F.C. (2016). Nicotinamide treatment in traumatic brain injury: Operation brain trauma therapy. J. Neurotrauma 33, 523-537.

3. Bramlett, H.M., Dietrich, W.D., Dixon, C.E., Shear, D.A., Schmid, K.E., Mondello, S., Wang, K.K., Hayes, R.L., Povlishock, J.T., Tortella, F.C., and Kochanek, P.M. (2016). Erythropoietin treatment in traumatic brain injury: Operation brain trauma therapy. J. Neurotrauma $33,538-552$.

4. Dixon, C.E., Bramlett, H.M., Dietrich, W.D., Shear, D.A., Yan, H.Q., Deng-Bryant, Y., Mondello, S., Wang, K.K., Hayes, R.L., Empey, P.E., Povlishock, J.T., Tortella, F.C., and Kochanek, P.M. (2016). Cyclosporine treatment in traumatic brain injury: Operation brain trauma therapy. J. Neurotrauma 33, 553-566.

5. Mountney, A., Bramlett, H.M., Dixon, C.E., Mondello, S., Dietrich, W.D., Wang, K.K.W., Hayes, R.L., Schmid, K.E., Povlishock, J.T., Tortella, F.C., Kochanek, P.M., and Shear, D.A. (2015). Simvastatin treatment in traumatic brain injury: Operation brain trauma therapy. J. Neurotrauma 33, 567-580.
6. Browning, M., Shear, D.A., Bramlett, H.M., Dixon, C.E., Mondello, S., Schmid, K.E., Poloyac, S.M., Dietrich, W.D., Hayes, R.L., Wang, K.K., Povlishock, J.T., Tortella, F.C., and Kochanek, P.M. (2016). Levetiracetam treatment in traumatic brain injury: Operation brain trauma therapy. J. Neurotrauma 33, 581-594.

7. Mondello, S., Shear, D.A., Bramlett, H.M., Dixon, C.E., Schmid, K.E., Dietrich, W.D., Wang, K. K., Hayes, R.L., Glushakova, O., Catania, M., Richieri, S., Povlishock, J.T., Tortella, F.C., and Kochanek, P.M. (2016). Insight into pre-clinical models of traumatic brain injury using circulating brain damage biomarkers: Operation brain trauma therapy. J. Neurotrauma 33, 595-605.

8 . Basso, D.M., Beattie, M.S., Bresnahan, J.C., Anderson, D.K., Faden, A.I., Gruner, J.A., Holford, T.R., Hsu, C.Y., Noble, L.J., Nockels, R., Perot, P.L., Salzman, S.K., and Young, W. (1996). MASCIS evaluation of open field locomotor scores: effects of experience and teamwork on reliability. Multicenter Animal Spinal Cord Injury Study. J. Neurotrauma 13, 343-359.

9. Beattie, M.S., Bresnahan, J.C., Komon, J., Tovar, C.A., Van Meter, M., Anderson, D.K., Faden, A.I., Hsu, C.Y., Noble, L.J., Salzman, S., and Young, W. (1997). Endogenous repair after spinal cord contusion injuries in the rat. Exp. Neurol. 148, 453-463.

10. Bigler, E.D. (2014). Comment: Importance of cognitive reserve in traumatic brain injury. Neurology 82, 1641.

11. Saatman, K.E., Duhaime, A.C., Bullock, R., Maas, A.I., Valadka, A., and Manley, G.T. (2008). Classification of traumatic brain injury for targeted therapies. J. Neurotrauma 25, 719-738.

12. Hawryluk, G.W.J., and Manley, G.T. (2015). Classification of traumatic brain injury: past, present, and future. Handb. Clin. Neurol. 127, $15-21$.

13. Papa, L., Lewis, L.M., Silvestri, S., Falk, J.L., Giordano, P., Brophy, G.M., Demery, J.A., Liu, M.C., Mo, J., Akinyi, L., Mondello, S., Schmid, K., Robertson, C.S., Tortella, F.C., Hayes, R.L., and Wang, K.K. (2012). Serum levels of ubiquitin C-terminal hydrolase distinguish mild traumatic brain injury from trauma controls and are elevated in mild and moderate traumatic brain injury patients with intracranial lesions and neurosurgical intervention. J. Trauma Acute Care Surg. 72, 1335-1344.

14. Plog, B.A., Dashnaw, M.L., Hitomi, E., Peng, W., Liao, Y., Lou, N., Deane, R., and Nedergaard, M. (2015). Biomarkers of traumatic injury are transported from brain to blood via the glymphatic system. J. Neurosci. 35, 518-526.

15. Wang, H., Gao, J., Lassiter, T.F., McDonagh, D.L., Sheng, H., Warner, D.S., Lynch, J.R., and Laskowitz, D.T. (2006). Levetiracetam is neuroprotective in murine models of closed head injury and subarachnoid hemorrhage. Neurocrit. Care 5, 71-78.

16. Hoane, M.R., Tan, A.A., Pierce, J.L., Anderson, G.D., and Smith, D.C. (2006). Nicotinamide treatment reduces behavioral impairments and provides cortical protection after fluid percussion injury in the rat. J. Neurotrauma 23, 1535-1548.

17. Hemerka, J.N., Wu, X., Dixon, C.E., Garman, R.H., Exo, J.L., Shellington, D.K., Blasiole, B., Vagni, V.A., Janesko-Feldman, K., Xu, M., Wisniewski, S.R., Bayir, H., Jenkins, L.W., Clark, R.S., Tisherman, S.A., and Kochanek, P.M. (2012). Severe brief pressure-controlled hemorrhagic shock after traumatic brain injury exacerbates functional deficits and long-term neuropathological damage in mice. J. Neurotrauma 29, 2192-2208.

18. Diaz-Arrastia, R., Kochanek, P.M., Bergold, P., Kenney, K., Marx, C.E., Grimes, C.J.B., Loh, L.T., Adam, L.T., Oskvig, D., Curley, K.C., and Salzer, W. (2014). Pharmacotherapy of traumatic brain injury: state of the science and the road forward: report of the Department of Defense Neurotrauma Pharmacology Workgroup. J. Neurotrauma 31, 135-158.

19. Robertson, C.S., Hannay, H.J., Yamal, J.-M., Gopinath, S., Goodman, J.C., Tilley, B.C.; Epo Severe TBI Investigators, Baldwin, A., Rivera Lara, L., Saucedo-Crespo, H., Ahmed, O., Sadasivan, S., Ponce, L., Cruz-Navarro, J., Shahin, H., Aisiku, I.P., Doshi, P., Valadka, A., Neipert, L., Waguspack, J.M., Rubin, M.L., Benoit, J.S., and Swank, P. (2014). Effect of erythropoietin and transfusion threshold on neurological recovery after traumatic brain injury: a randomized clinical trial. JAMA 312, 36-47.

20. Ehrenreich, H., Weissenborn, K., Prange, H., Schneider, D., Weimar, C., Wartenberg, K., Schellinger, P.D., Bohn, M., Becker, H., Wegrzyn, M., Jähnig, P., Herrmann, M., Knauth, M., Bähr, M., Heide, W., Wagner, A., Schwab, S., Reichmann, H., Schwendemann, G., Dengler, R., Kastrup, A., and Bartels, C. (2009). Recombinant human erythropoietin in the treatment of acute ischemic stroke. Stroke 40, e647-e656. 
21. Begley, C.G., and Ellis, L.M. (2012). Drug development: Raise standards for preclinical cancer research. Nature 483, 531-533.

22. Begley, C.G., and Ioannidis, J.P. (2015). Reproducibility in science: improving the standard for basic and preclinical research. Circ. Res. $116,116-126$.

23. Lapchak, P.A., Zhang, J.H., and Noble-Haeusslein, L.J. (2013). RIGOR guidelines: escalating STAIR and STEPS for effective translational research. Transl. Stroke Res. 4, 279-285.

24. Reier, P.J., Lane, M.A., Hall, E.D., Teng, Y.D., and Howland, D.R. (2012). Translational spinal cord injury research: preclinical guidelines and challenges. Handb. Clin. Neurol. 109, 411-433.

25. Warner, D.S., James, M.L., Laskowitz, D.T., and Wijdicks, E.F. (2014). Translational research in acute central nervous system injury: lessons learned and the future. JAMA Neurol. 71, 1311-1318.

26. Smith, D.H., Hicks, R.R., Johnson, V.E., Bergstrom, D.A., Cummings, D.M., Noble, L.J., Hovda, D., Whalen, M.J., Ahlers, S.T., LaPlaca, M., Tortella, F.C., Duhaime, A.C., and Dixon, C.E. (2015). Preclinical traumatic brain injury common data elements: toward a common language across laboratories. J. Neurotrauma 32, 1725-1735.

27. Garman, R.H., Jenkins, L.W., Switzer, R.C., 3rd, Bauman, R.A., Tong, L.C., Swauger, P. V, Parks, S.A., Ritzel, D. V, Dixon, C.E., Clark, R.S., Bayir, H., Kagan, V., Jackson, E.K., and Kochanek, P.M (2011). Blast exposure in rats with body shielding is characterized primarily by diffuse axonal injury. J. Neurotrauma 28, 947-959.

28. DeWitt, D.S., and Prough, D.S. (2009). Blast-induced brain injury and posttraumatic hypotension and hypoxemia. J. Neurotrauma 26, 877-887.

29. Shein, S.L., Shellington, D.K., Exo, J.L., Jackson, T.C., Wisniewski, S.R., Jackson, E.K., Vagni, V.A., Bayir, H., Clark, R.S., Dixon, C.E., Janesko-Feldman, K.L., and Kochanek, P.M. (2014). Hemorrhagic shock shifts the serum cytokine profile from pro- to anti-inflammatory after experimental traumatic brain injury in mice. J. Neurotrauma 31 , 1386-1395.

30. Morganti-Kossmann, M.C., Yan, E., and Bye, N. (2010). Animal models of traumatic brain injury: is there an optimal model to reproduce human brain injury in the laboratory? Injury 41, Suppl 1, S10-S13.

31. Foda, M.A., and Marmarou, A. (1994). A new model of diffuse brain injury in rats. Part II: morphological characterization. J. Neurosurg. $80,301-313$

32. Goldstein, L.E., Fisher, A.M., Tagge, C.A., Zhang, X.-L., Velisek, L., Sullivan, J.A., Upreti, C., Kracht, J.M., Ericsson, M., Wojnarowicz, M.W., Goletiani, C.J., Maglakelidze, G.M., Casey, N., Moncaster, J.A., Minaeva, O., Moir, R.D., Nowinski, C.J., Stern, R.A., Cantu, R.C., Geiling, J., Blusztajn, J.K., Wolozin, B.L., Ikezu, T., Stein, T.D., Budson, A.E., Kowall, N.W., Chargin, D., Sharon, A., Saman, S., Hall, G.F., Moss, W.C., Cleveland, R.O., Tanzi, R.E., Stanton, P.K., and McKee, A.C. (2012). Chronic traumatic encephalopathy in blastexposed military veterans and a blast neurotrauma mouse model. Sci. Transl. Med. 4, 134ra60.

33. Statler, K.D., Jenkins, L.W., Dixon, C.E., Clark, R.S., Marion, D.W., and Kochanek, P.M. (2001). The simple model versus the super model: translating experimental traumatic brain injury research to the bedside. J. Neurotrauma 18, 1195-1206.

34. Wright, D.W., Yeatts, S.D., Silbergleit, R., Palesch, Y.Y., Hertzberg, V.S., Frankel, M., Goldstein, F.C., Caveney, A.F., Howlett-Smith, H., Bengelink, E.M., Manley, G.T., Merck, L.H., Janis, L.S., Barsan, W.G., and NETT Investigators. (2014). Very early administration of progesterone for acute traumatic brain injury. N. Engl. J. Med. 371, 2457-2466.

35. Bullock, M.R., Lyeth, B.G., and Muizelaar, J.P. (1999). Current status of neuroprotection trials for traumatic brain injury: lessons from animal models and clinical studies. Neurosurgery 45, 207-220.

36. Tolias, C.M., and Bullock, M.R. (2004). Critical appraisal of neuroprotection trials in head injury: what have we learned? NeuroRx 1, 71-79.

37. Darrah, S.D., Chuang, J., Mohler, L.M., Chen, X., Cummings, E.E., Burnett, T., Reyes-Littaua, M.C., Galang, G.N., and Wagner, A.K. (2011). Dilantin therapy in an experimental model of traumatic brain injury: effects of limited versus daily treatment on neurological and behavioral recovery. J. Neurotrauma 28, 43-55.

38. Simard, J.M., Tsymbalyuk, N., Tsymbalyuk, O., Ivanova, S., Yurovsky, V., and Gerzanich, V. (2010). Glibenclamide is superior to decompressive craniectomy in a rat model of malignant stroke. Stroke. 41, 531-537.
39. Bye, N., Habgood, M.D., Callaway, J.K., Malakooti, N., Potter, A., Kossmann, T., and Morganti-Kossmann, M.C. (2007). Transient neuroprotection by minocycline following traumatic brain injury is associated with attenuated microglial activation but no changes in cell apoptosis or neutrophil infiltration. Exp. Neurol. 204, 220-233.

40. Haber, M., Abdel Baki, S.G., Grin'kina, N.M., Irizarry, R., Ershova, A., Orsi, S., Grill, R.J., Dash, P., and Bergold, P.J. (2013). Minocycline plus $\mathrm{N}$-acetylcysteine synergize to modulate inflammation and prevent cognitive and memory deficits in a rat model of mild traumatic brain injury. Exp. Neurol. 249, 169-177.

41. Dixon, C.E., Kraus, M.F., Kline, A.E., Ma, X., Yan, H.Q., Griffith, R.G., Wolfson, B.M., and Marion, D.W. (1999). Amantadine improves water maze performance without affecting motor behavior following traumatic brain injury in rats. Restor. Neurol. Neurosci. 14, 285-294.

42. Wang, T., Huang, X.J., Van, K.C., Went, G.T., Nguyen, J.T., and Lyeth, B.G. (2014). Amantadine improves cognitive outcome and increases neuronal survival after fluid percussion traumatic brain injury in rats. J. Neurotrauma 31, 370-377.

43. Giacino, J.T., Whyte, J., Bagiella, E., Kalmar, K., Childs, N., Khademi, A., Eifert, B., Long, D., Katz, D.I., Cho, S., Yablon, S.A., Luther, M., Hammond, F.M., Nordenbo, A., Novak, P., Mercer, W., Maurer-Karattup, P., and Sherer, M. (2012). Placebo-controlled trial of amantadine for severe traumatic brain injury. N. Engl. J. Med. 366, 819-826.

44. Kochanek, P.M., Jackson, T.C., Ferguson, N.M., Carlson, S.W., Simon, D.W., Brockman, E.C., Ji, J., Bayir, H., Poloyac, S.M., Wagner, A.K., Kline, A.E., Empey, P.E., Clark, R.S., Jackson, E.K., and Dixon, C.E. (2015). Emerging therapies in traumatic brain injury. Semin. Neurol. 35, 83-100.

45. Liao, G.P., Harting, M.T., Hetz, R.A., Walker, P.A., Shah, S.K., Corkins, C.J., Hughes, T.G., Jimenez, F., Kosmach, S.C., Day, M.C., Tsao, K., Lee, D.A., Worth, L.L., Baumgartner, J.E., and Cox, C.S., Jr. (2015). Autologous bone marrow mononuclear cells reduce therapeutic intensity for severe traumatic brain injury in children. Pediatr. Crit. Care Med. 16, 245-255.

46. Kernie, S.G. (2015). Cell-based therapy for pediatric traumatic brain injury: not (yet) an update to the traumatic brain injury guidelines. Pediatr. Crit. Care Med. 16, 294-295.

47. Khuman, J., Zhang, J., Park, J., Carroll, J.D., Donahue, C., and Whalen, M.J. (2012). Low-level laser light therapy improves cognitive deficits and inhibits microglial activation after controlled cortical impact in mice. J. Neurotrauma 29, 408-417.

48. Loane, D.J., Kumar, A., Stoica, B.A., Cabatbat, R., and Faden, A.I. (2014). Progressive neurodegeneration after experimental brain trauma: association with chronic microglial activation. J. Neuropathol. Exp. Neurol. 73, 14-29.

49. Kovesdi, E., Kamnaksh, A., Wingo, D., Ahmed, F., Grunberg, N.E., Long, J.B., Kasper, C.E., and Agoston, D.V. (2012). Acute minocycline treatment mitigates the symptoms of mild blast-induced traumatic brain injury. Front. Neurol. 3, 111.

50. Dixon, C.E., Kochanek, P.M., Yan, H.Q., Schiding, J.K., Griffith, R.G., Baum, E., Marion, D.W., and DeKosky, S.T. (1999). One-year study of spatial memory performance, brain morphology, and cholinergic markers after moderate controlled cortical impact in rats. J. Neurotrauma 16, 109-122.

51. Bramlett, H.M., and Dietrich, W.D. (2002). Quantitative structural changes in white and gray matter 1 year following traumatic brain injury in rats. Acta Neuropathol. 103, 607-614.

52. Bramlett, H.M., and Dietrich, W.D. (2014). Long-term consequences of traumatic brain injury: current status of potential mechanisms of injury and neurological outcomes. J. Neurotrauma. 32, 1834-1848.

Address correspondence to: Patrick M. Kochanek, MD, MCCM Department of Critical Care Medicine Safar Center for Resuscitation Research University of Pittsburgh School of Medicine 3434 Fifth Avenue Pittsburgh, PA 15260

E-mail: kochanekpm@ccm.upmc.edu 